\title{
Engineering monitoring of rockfall hazards along transportation corridors: using mobile terrestrial LiDAR
}

\author{
M. Lato ${ }^{1}$, J. Hutchinson ${ }^{1}$, M. Diederichs ${ }^{1}$, D. Ball ${ }^{2}$, and R. Harrap ${ }^{1}$ \\ ${ }^{1}$ GeoEngineering Centre, Queen's University at Kingston, On., Canada \\ ${ }^{2}$ Geological Sciences and Geological Engineering, Queen's University at Kingston, On., Canada
}

Received: 2 December 2008 - Revised: 27 May 2009 - Accepted: 8 June 2009 - Published: 23 June 2009

\begin{abstract}
Geotechnical hazards along linear transportation corridors are challenging to identify and often require constant monitoring. Inspecting corridors using traditional, manual methods requires the engineer to be unnecessarily exposed to the hazard. It also requires closure of the corridor to ensure safety of the worker from passing vehicles. This paper identifies the use of mobile terrestrial LiDAR data as a compliment to traditional field methods. Mobile terrestrial LiDAR is an emerging remote data collection technique capable of generating accurate fully three-dimensional virtual models while driving at speeds up to $100 \mathrm{~km} / \mathrm{h}$. Data is collected from a truck that causes no delays to active traffic nor does it impede corridor use. These resultant georeferenced data can be used for geomechanical structural feature identification and kinematic analysis, rockfall path identification and differential monitoring of rock movement or failure over time. Comparisons between mobile terrestrial and static LiDAR data collection and analysis are presented. As well, detailed discussions on workflow procedures for possible implementation are discussed. Future use of mobile terrestrial LiDAR data for corridor analysis will focus on repeated surveys and developing dynamic four-dimensional models, higher resolution data collection. As well, computationally advanced, spatially accurate, geomechanically controlled three-dimensional rockfall simulations should be investigated.
\end{abstract}

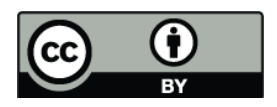

Correspondence to: M. Lato

(mjlato@gmail.com)

\section{Introduction}

Geotechnical hazards, especially rockfall related hazards, pose a significant concern to the management of transportation corridors (Maerz, 2000). Extensive rail and road networks in regions of rugged terrain, such as the Alps, the Superior Province of Canada, and the Canadian Cordillera, have complex relationships with their geological surroundings, and yet carry significant traffic. For operators of transportation corridors, the ability to identify potential hazards, assess geomechanics parameters alongside the track /road and measure movement, while maintaining safety for both traffic and workers, is paramount. This paper examines new techniques for geomechanical evaluation along transportation corridors. Three site locations within Ontario, Canada were used for the analyses: two sections of Provincial Highway 15 near Ottawa, and a section of track along the Algoma Central Railway (ACR) in Northern Ontario.

A typical hazard assessment flowchart for engineered monitoring of rockcuts and slopes is shown in Fig. 1. Variables on the left hand side illustrate hazard values that can be directly or indirectly determined. The flowchart assumes that the onsite or overseeing engineers are aware of potentially unstable and hazardous rockfall locations. This flowchart forms a foundation for in-the-field and in-office workflows for the assessment of hazards and thus forms a first-step in planning hazard mitigation. We herein examine use of light detection and ranging - LiDAR, to help manage and assess rockfall hazards as a specific case in linear infrastructure management; LiDAR represents a new approach to contributing to the geomechanical workflow.

Traditionally, LiDAR units are statically mounted on tripods or deployed for mobile applications in helicopters and airplanes. Aerial LiDAR employs similar scanning devices to static equipment but are additionally equipped with

Published by Copernicus Publications on behalf of the European Geosciences Union. 


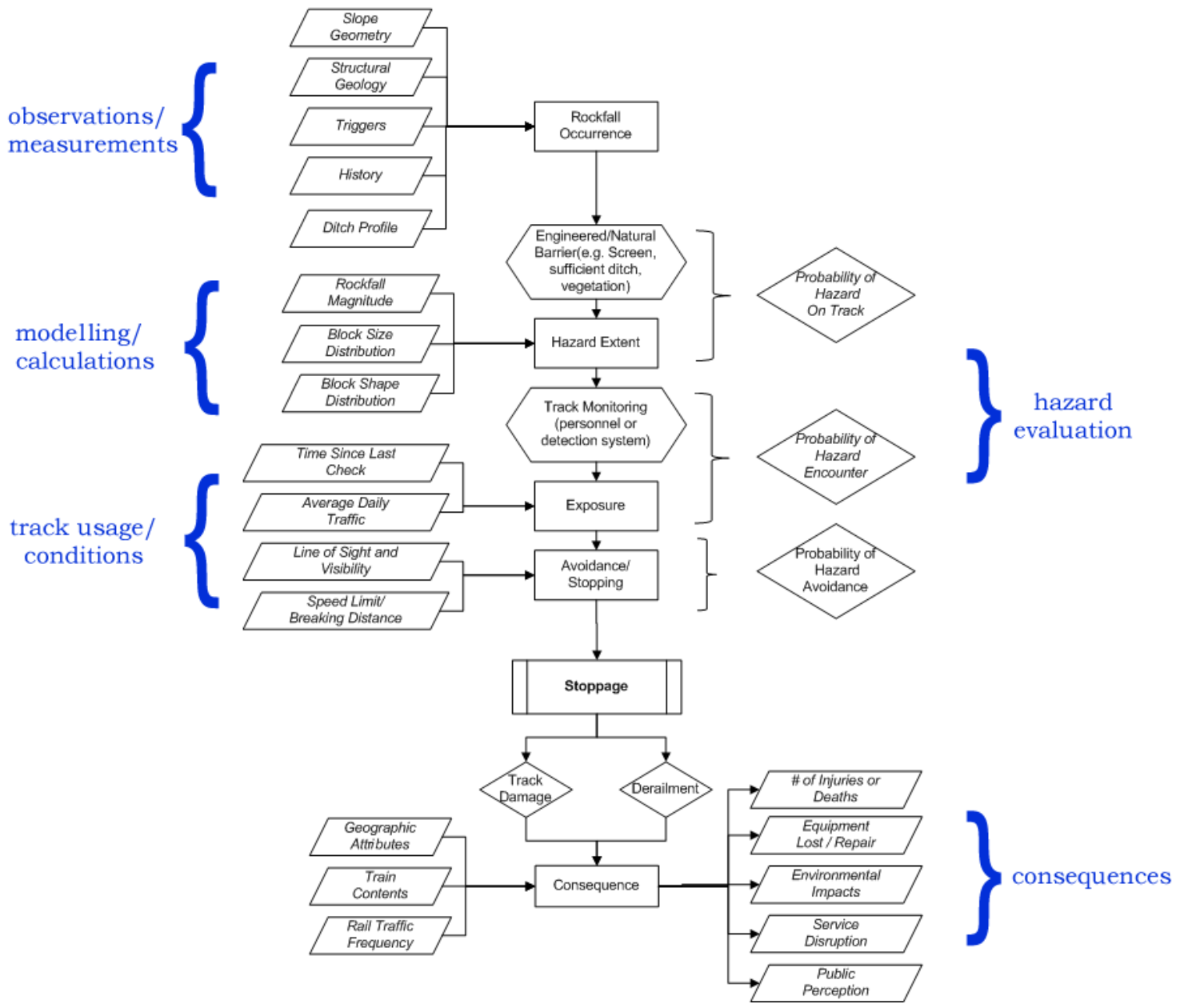

Fig. 1. Engineered monitoring of rockcuts and slopes, specifically along railway corridors. Information measured, modeled, or calculated are displayed as inputs on the left hand side. Probabilities are calculated based on discrete inputs.

a global positioning system (GPS) and inertial navigation systems (INS, also known as an inertial measurement unit - IMU) to determine location during flight (Yang and Farrell, 2003). Through recent innovations, static LiDAR sensors have been deployed in truck mounted mobile terrestrial applications (Glennie, 2007a).

Mobile terrestrial LiDAR is a breakthrough technology for the field of geotechnical engineering that can be used effectively within a rockfall hazard management system, LiDAR data, in the form of a point-cloud, can be collected for structurally related geomechanical evaluation purposes at speeds of up to $30 \mathrm{~km} / \mathrm{h}$. At this rate, data can be quickly collected for large corridors of track or roadways. Employing mobile terrestrial LiDAR enables the rapid development of an accurate spatially inclusive LiDAR dataset for many geotechnical stability issues. This LiDAR data can subsequently be used for accurate spatial identification of track features such as rails, crossings, and signs as well as temporal differential modelling.
The baseline dataset can be used to develop a 3dimensional (3-D) model of the corridor. This computer based geometric model will aid in developing GIS maps and digital elevation models (DEM), the identification of potential hazardous locations, and the ability to compare and differentiate the baseline data against subsequent temporal scans.

The LiDAR-based model, in conjunction with active track supervision by engineers, provides a valuable rockfall evaluation tool (Turner, et al., 2006; Rosser et al., 2005). The engineers currently monitoring the ACR, for example, use a customized rockfall hazard rating assessment system $(\mathrm{Ab}$ bott, et al., 1998) based on field observations. This system aids in categorizing and rating potential hazards based on geomechanics, track configuration, and slope properties. Identifying zones along the track corridor that are perceived to be hazardous either because of visible signs of failure or because of a high rockfall hazard rating as denoted by the assessment, further remote investigation can be completed using the baseline LiDAR dataset (Sturzenegger et al., 2007a). 


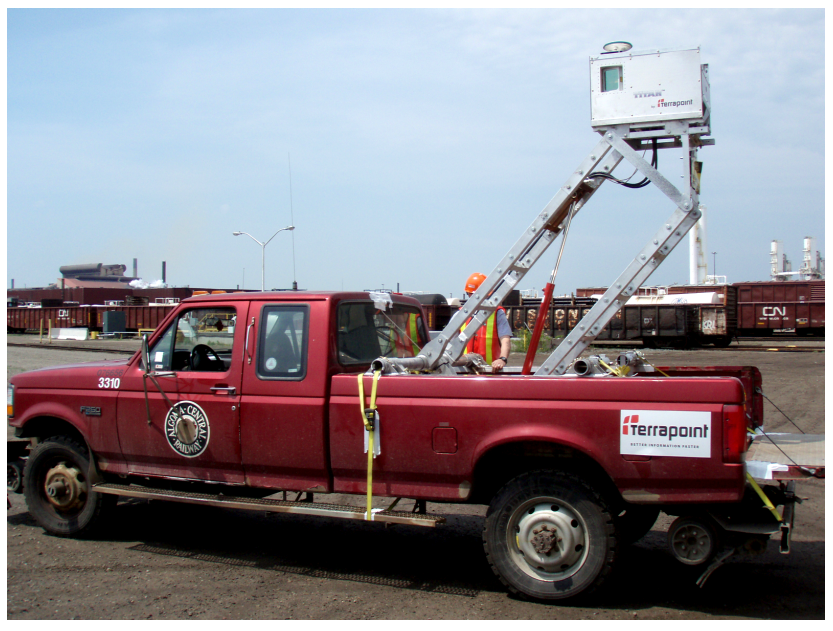

Fig. 2. The TITAN system (silver box) is a multi-LiDAR scanner. Here is it shown mounted in the bed of a high-rail truck for deployment along the Algoma Central Railway.

The information that can be extracted from LiDAR data includes detailed geometry of the rock faces, track, ditches, other infrastructure, structural geological mapping and characterization, and evaluation of the vehicle operator's lineof-sight. Comparison of data from subsequent scans allows evaluation of rock deformation and volumetric change. Baseline LiDAR data provides information which is required for an improved hazard management workflow: more efficient, repeatable, and reproducible.

LiDAR data has been used for many geologically related academic and industry projects over the last ten years. The projects have ranged from landslide detection (McKean and Roering, 2004) to debris flow modeling (Staley et al., 2005) and flood predictions (Mason et al., 2007).

\subsection{Mobile terrestrial LiDAR}

Mobile terrestrial LiDAR systems are comprised of a vehicle, a network of LiDAR sensors, a carrier-phase differential GPS network, and an inertial navigation system (INS). The GPS network employed for this operation consisted of one onboard receiver, one static receiver located in the centre of the scanning area (a maximum distance of $10 \mathrm{~km}$ from the TITAN truck), and two static receivers located over federally established benchmarks. The result of a scan from such a system is a cloud of 3-D positions, reported in a geographic coordinate system. This point cloud has several important characteristics:

- Points are limited to features visible from the perspective of the scanner (it is a line-of-sight technology).

- Point density decreases as distance from the scanner increases; the sensor has a maximum range of $200 \mathrm{~m}$.

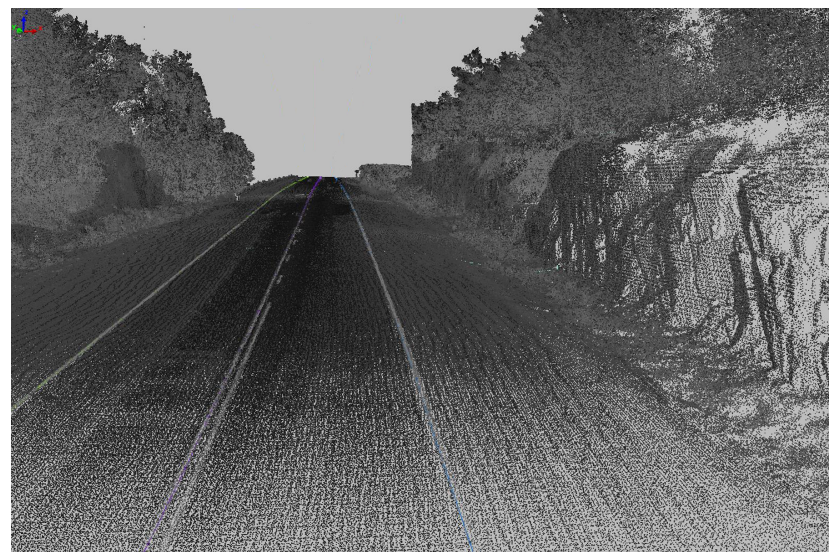

Fig. 3. LiDAR data collected by the TITAN system. Note for scale the rockmass present in the right-hand side of the cloud is roughly $5 \mathrm{~m}$ tall. This data was collected near Ottawa, Ontario, June 2007.

- Points are collected at a high rate (TITAN, collects at $40000 \mathrm{pts} / \mathrm{s})$.

- The strength of the return is measured and reported as an intensity value, which allows genuine visual discrimination of different materials.

The mobile terrestrial scanner used for this specific application was TITAN, developed by Terrapoint, a division of Ambercore (Glennie, 2007a). The TITAN system (as shown in Fig. 2) is mounted on a pickup truck. A truck capable of traveling on a rail track was used for scanning along the ACR. The silver pod elevated above the bed of the truck contains four independent, uniquely oriented LiDAR scanning units, one global positioning system (GPS) antenna, and one INS system. The elevation of the pod is controlled from within the operator's cabin by a hydraulic lift. Other mobile terrestrial LiDAR systems include the Lynx (Optech, 2008) and active research projects by Alshawa et al. (2007).

A typical LiDAR point cloud generated from the TITAN mobile system is displayed in Fig. 3. While this appears to be a grey-scale photograph, it is actually a view of tens of millions of 3-D points surveyed across a rockmass face and highway surface. Recorded intensity values allow visible discrimination of painted lines on the road surface due to the materials reflective properties.

Regardless of the sensor technology, in a mobile scanning configuration, the position of the scanner at the instant of scanning must be known. This is accomplished by coupling a GPS receiver and an INS. GPS receivers accurately determine their position by time-of-flight determination to multiple satellites from a roving field sensor (El-Rabbany, 2006). For precise mobile terrestrial applications, carrier phase GPS is used, which enables sub $5 \mathrm{~cm}$ positioning. It is essential because the final positional accuracy of scan points relies on both scanner precision and the accuracy of the scanner pod position. 


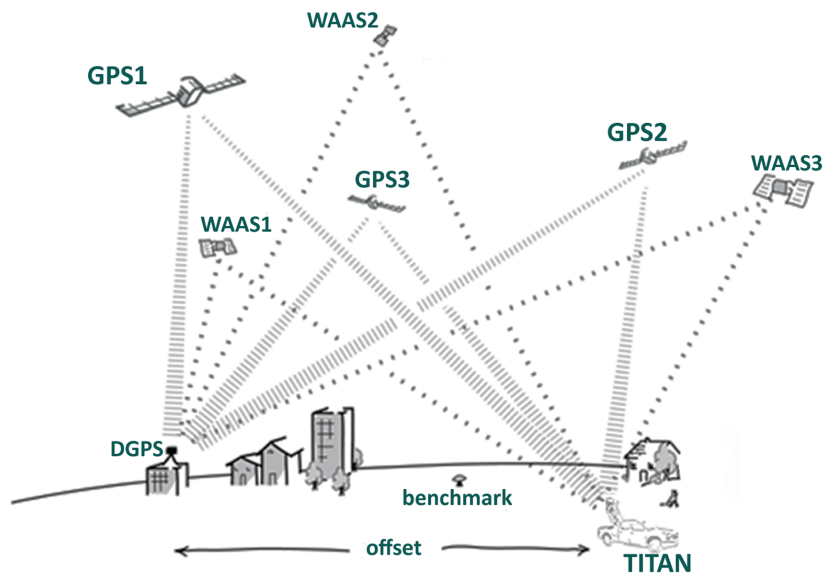

Fig. 4. Differential GPS data collection as employed by the TITAN system. The system is comprised of a network of satellites and ground receivers.

Due to the vibration and movement of the vehicle, complex movements of millimetres and centimetres per second are superimposed on the overall vehicle path. As a result, a simple interpolation between GPS positions (collected once per second) is inadequate for properly positioning each LiDAR data point. An INS, consisting of coupled gyroscopes and accelerometers, is used to solve this problem, and, in addition, the INS also provides path reconstruction during short intervals when GPS is unavailable due to obstructed views of the sky (Yang and Farrell, 2006). Importantly, the presence of an INS means that TITAN is capable of scanning short tunnels and underpasses or canyons without loss of precision. The relative roles of GPS and INS are illustrated in Figs. 4 and 5.

Globally referenced LiDAR data inherently has two associated accuracy values, commonly known as local and global accuracy. Local accuracy is primarily a factor of sensor error - which is the observed error of the LiDAR sensor itself. For TITAN data, this error is roughly $1 \mathrm{~cm}$ per $100 \mathrm{~m}$ distance between the object and the sensor. Global accuracy is dependent on the GPS-INS solution as well as the LiDAR sensor error, and is a measure of how well the determined locations of 3-D points match their true geographic locations. Global accuracy decreases as the distance from the differential GPS base station increases, at a maximum rate of $0.5 \mathrm{~cm}$ per $1 \mathrm{~km}$ with an initial error of $5 \mathrm{~mm}$; this is calculated at $0.5 \mathrm{~cm}+5 \mathrm{ppm}$ (Glennie, 2007b). A general but thorough overview of accuracy considerations for mobile terrestrial mapping was conducted and published by Barber et al. (2008). Global accuracy does not affect the viewability or the analysis of the data, only the relation of the identified features to their true locations in space, and so to other GIS datasets. It is essential for the geotechnical use of this data that the data collection errors be minimized. If the data are going to be used for orientation-based measurements

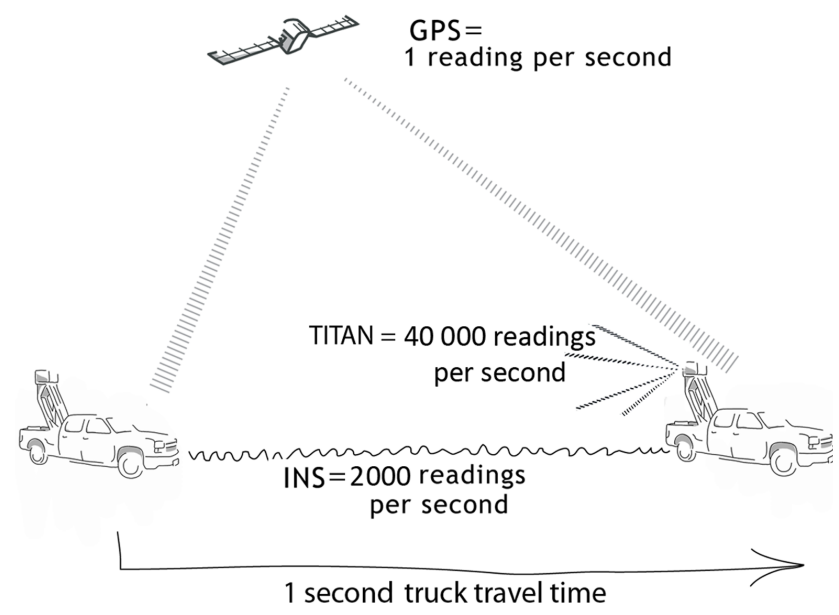

Fig. 5. GPS - INS - LIDAR sensors work in concert to produce globally registered and locally accurate point clouds.

the errors will propagate through subsequent analyses as discussed by Buckley et al. (2008).

\subsection{Mobile terrestrial LiDAR data and static data}

Conventionally, LiDAR data is collected from static platforms such as tripods or from mobile aerial vehicles. Data densities range from $10000 \mathrm{pts} / \mathrm{m}^{2}$ for static systems and down to $10 \mathrm{pts} / \mathrm{m}^{2}$ for aerial systems. TITAN employs static scanning technology (via Riegl sensors) on a mobile (truck) platform. The resultant data densities practically range from 50 to $500 \mathrm{pts} / \mathrm{m}^{2}$ (depending on velocity). Figure 6 illustrates a direct comparison of data collected with a static Leica HDS 6000 system and the TITAN system. The Leica HDS 6000 collects up to $500000 \mathrm{pts} / \mathrm{s}$ at a density of up to $10000 \mathrm{pts} / \mathrm{m}^{2}$. Figure 6 also illustrates the cross-sectional continuity of the datasets and the ability to combine data from separate scans to increase viewability while minimizing occlusion, which are zones of no data due to obstruction.

Figure 6 illustrates that the data collected by the TITAN system displays slightly greater local noise in comparison to the Leica data; as well it is more challenging to visualize due to the reduced point density. However, it is sufficient to perform accurate geomechanical structural analyses as will be demonstrated below. Data are processed using PolyWorks (InnovMetric, 2008).

\subsection{Feature extraction and inventory from LiDAR data}

Spatially accurate LiDAR data can be used to semiautomatically measure physical features typically measured by hand in the field. From a geotechnical perspective, measurements that can be rapidly acquired from LiDAR data include: slope angle and geometry, ditch height, width, and angle, and track geometry parameters such as curvature. As well, geomechanical structural data can be assessed in terms 


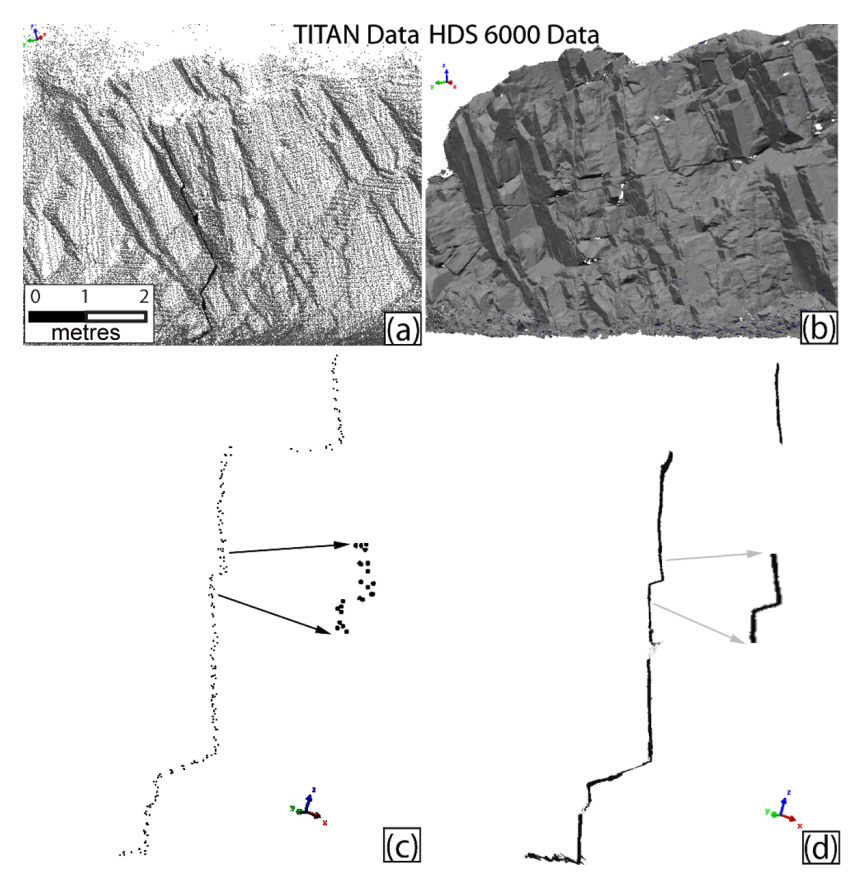

Fig. 6. (a) TITAN data collected in Ottawa, Ontario, June 2007, for an outcrop of well-fractured granite (b) illustrates the same outcrop scanned with a Leica HDS 6000. (c) and (d) illustrate a crosssection of the collected data from (a) and (b) respectfully.

of discontinuity surface orientation and spacing (Feng and Roshoff, 2004). The advantage of extracting these features from LiDAR data is that locations of measured features are automatically generated because the data has inherent spatial attributes, there is increased precision of the measurements, direct digital storage, and there is the ability to review measurements. As well, the collection of this data does not involve the closure or obstruction of the track due to engineering personnel being present on the track; instead the data is quickly collected from a mobile platform and processed off-site - which increases the safety of the engineer as a result.

\section{Algoma railway LiDAR project}

The specific field test of the use of TITAN for geomechanical evaluation was carried out along the Algoma Central Railway, with the collaboration of CN Rail, Transport Canada, Terrapoint and Queen's University.

\subsection{Project background}

The Algoma Central Railway (ACR) was commissioned for construction in early 1899 and is currently owned and operated by Canadian National Railway (CN). It is currently used for industrial (logging) and transportation/tourism purposes. The ACR is $475 \mathrm{~km}$ long and extends from Sault Ste. Marie,

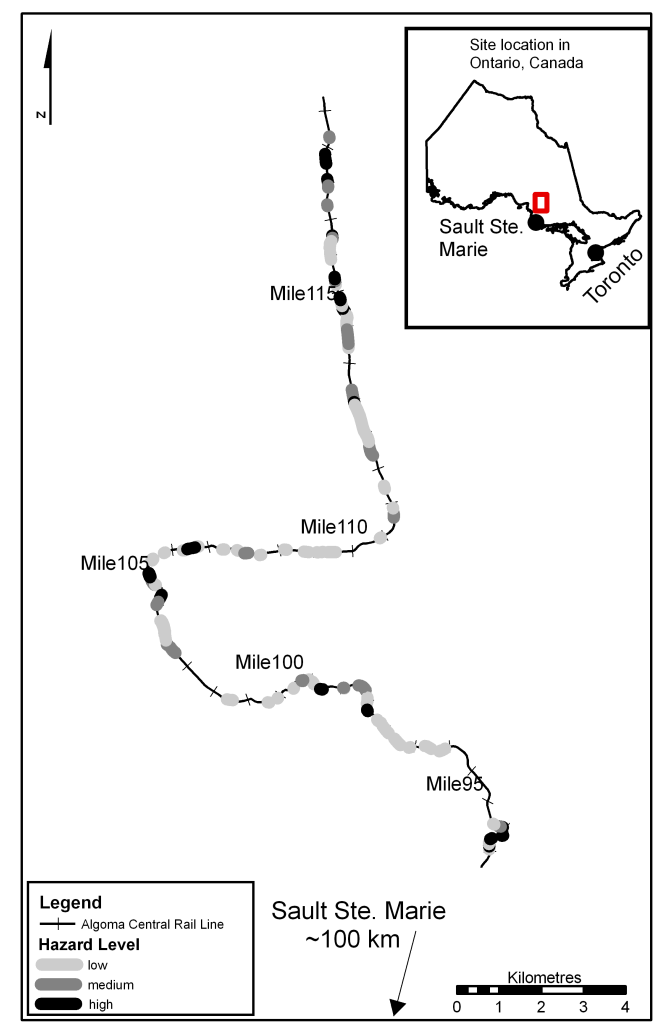

Fig. 7. Helicopter and TITAN LiDAR data were collected along the Algoma Central Railway in July 2007 and June 2008 respectively.

Ontario to Hearst, Ontario. The ACR runs along the banks of the Algoma River, the course of which is controlled by a Northeast-Southwest trending strike slip fault. The valley ridges range in elevation up to $900 \mathrm{~m}$ above the track level.

Blasting and excavation techniques employed in construction in the early 1900's were much less sophisticated than modern methods. The combination of primitive blasting with 100 years of weathering and track operation has resulted in numerous sites that pose significant rockfall hazards to passing trains and their riders. $\mathrm{CN}$, with the assistance of Bruce Geotechnical Consultants (BGC), have mapped and evaluated all potential hazard locations along the ACR according to methods developed by BGC (Pritchard et al., 2005). The result of the mapping project is a Rockfall Hazard Rating Assessment (RHRA) value for each location identified as potentially hazardous. The main challenge of this system is the ability of the engineer to accurately measure variables such as slope height, angle, and profile without being able to physically climb the face. As well, measurements pertaining to the rockmass itself (structure orientation) are often generalized based on a random sampling of a few visible features. Figure 7 illustrates a section of the ACR that was mapped using the BGC methodology and displays the assessed RHRA values. This section of track was subsequently scanned with TITAN and helicopter LiDAR systems. As a result of the 

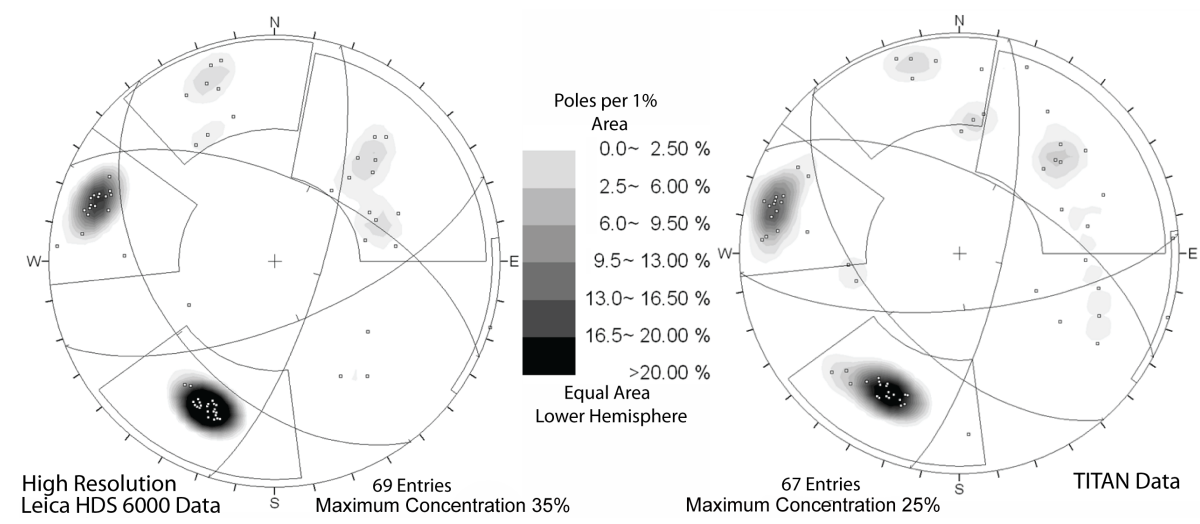

Fig. 8. Manual identification of structural features in LiDAR data using PolyWorks. These stereonets show a comparison between mapping using high resolution Leica HDS 6000 data and lower-resolution TITAN data. Results indicate a strong agreement between the data sets.

multiple LiDAR datasets and detailed human mapping and analysis, this section of track is an excellent case history site to assess how LiDAR can augment the data collected by a skilled engineer.

The scanning of the ACR with TITAN was an innovative use of both a high-rail truck (a truck designed to drive on rail tracks) and mobile terrestrial LiDAR equipment. The data collection did not impede or disrupt scheduled traffic along the ACR. The TITAN data were collected along the $20 \mathrm{~km}$ stretch of the ACR illustrated in Fig. 7. The total collection time was $5 \mathrm{~h}$, including $3.5 \mathrm{~h}$ for deployment.

The rate of LiDAR data collection from TITAN is $40000 \mathrm{pts} / \mathrm{s}-$ a measure that is independent of truck speed. In order to produce data of sufficient density to extract discrete geological features, sections of track known to be of high rockfall hazard potential, based on ratings determined using the BGC system, were driven at a slower speed than the non-hazardous locations along the ACR.

The most significant operational issue encountered while planning and collecting the data was quality of GPS signals. Due to the location of the ACR within a canyon, sky visibility is typically poor. Using high sampling and high precision INS alleviated this limitation. The global accuracy of the TITAN data collected along the ACR is $\pm 15 \mathrm{~cm}$ and the local accuracy of the data for any given section is approximately $\pm 3 \mathrm{~cm}$. Following collection, data was delivered in a time stamped globally referenced X, Y, Z, I point-cloud format. The file size is $15 \mathrm{~Gb}$.

\section{Mobile terrestrial feature extraction}

Mobile terrestrial LiDAR data collected by TITAN can be used to measure features such as joints and fractures. However, before the measurements can be used for engineering evaluation and design, the accuracy of the extracted features must be assessed. In order to achieve this, a reference roadcut (in the Ottawa area) was scanned using both the TITAN and the Leica HDS 6000 LiDAR systems. The results of this evaluation are illustrated in Fig. 8. Previous work by Lato et al. (2009) has demonstrated that the use of data generated by static LiDAR equipment is viable for geomechanical evaluation of structural features. The data were evaluated using Dips (RocScience, 2006), a stereographic projection analysis tool. As can be seen in Fig. 8, the data from mobile and static sources is compatible.

The processing workflow for structural features measured from both the TITAN data and the Leica HDS 6000 were completed using PolyWorks software, a state-of-the-art LiDAR processing tool. All planes were identified in the same manual manner to avoid user bias as recommended by Sturzenegger et al. (2007b). The extraction time per feature is roughly $20 \mathrm{~s}$, allowing an individual rockfall zone to be structurally evaluated in roughly $15-20 \mathrm{~min}$. To facilitate this process a macro was written in PolyWorks to automate the creation of a plane based on selected points by the user that visually lie on the same structural surface. The minimum area of a joint detectable is dependent on the density of the LiDAR data. As data density increases, smaller features become apparent and their orientations can be extracted. The density of the TITAN data in regions of interest along the ACR is roughly $500 \mathrm{pts} / \mathrm{m}^{2}$ and the minimum discontinuity size detectable is thus roughly $20 \mathrm{~cm} \times 20 \mathrm{~cm}$. The ability to identify a discontinuity surface depends on both its size and orientation with respect to the scanning equipment. As the angle between the scanner and the surface increases toward $90^{\circ}$ the feature is occluded from the point cloud. There are currently no mathematical solutions to resolve this issue, so care is taken with evaluation of the LiDAR data and time should be reserved to explore the data to look for structures at suspect orientations.

Once the LiDAR data has been evaluated for structural features, the orientation of the measured surface is converted 


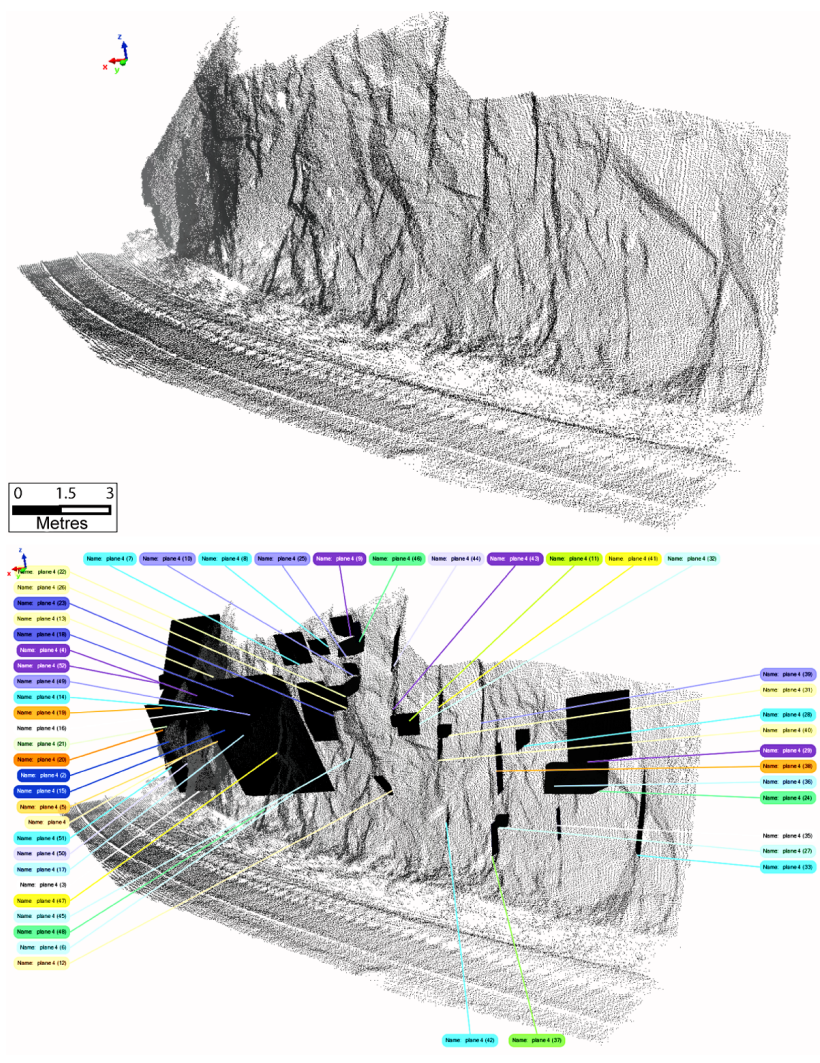

Fig. 9. Geomechanical feature identification and plane extraction of TITAN data collected along the Algoma Central Railway. Callout tabs represent each identified planar feature identified in the selected segment of track.

from geographic $\boldsymbol{i}, \boldsymbol{j}, \boldsymbol{k}$ unit vectors (vectors representing the surface oriented with respect to a global coordinate system) to dip and dip direction. This process is automatically conducted using a macro in MS Excel. The generated orientations are then analyzed using Dips. Herein joint sets can be identified, orientations can be evaluated, and potential failure modes can be determined.

\subsection{Mile 94: test site workflow}

Mile 94 on the ACR was identified using the BGC RHRA evaluation system as a high hazard zone. As shown in Fig. 9, the rockmass is near vertical, parallel to the track and ranges in height from $10-15 \mathrm{~m}$ above track level. The slope face ranges from an angle of $70^{\circ}$ to slightly overhanging. A detailed structural evaluation was completed using PolyWorks to determine the orientation of all visible planar discontinuities as scanned by TITAN. 52 joint surfaces were identified, measured and evaluated using the method described above.

The structural features identified in Fig. 9, were converted to dip and dip direction and analyzed in Dips. Joint sets and mean orientations were identified as illustrated in Fig. 10.

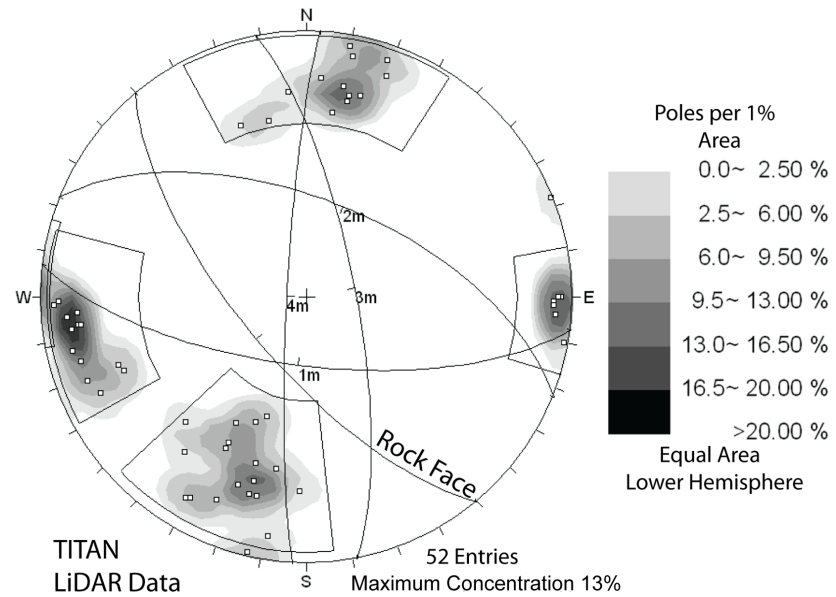

Fig. 10. Stereonet illustrating the geomechanical features extracted from TITAN data collected near mile 94 of the Algoma Central Railway. The rock face dips between $70^{\circ}$ to slightly overhanging (in this image and subsequent images it is illustrated at $70^{\circ}$ ).

As determined by visual inspection and using Dips, there are three main joint sets present in this rockcut.

Upon completion of the structural data extraction and evaluation based on track location and orientation with respect to the outcrop the rockcut can be evaluated for potential kinematic failure modes. Along the evaluated section of Mile 94 the rockmass is prone to both toppling and wedge failure modes, as illustrated in Fig. 11.

Toppling failure occurs when the dip of a joint exceeds the angle of the slope of the face by the friction angle of the rockmass (Goodman, 1980). The strike of the joint must be within $30^{\circ}$ of parallel to the strike of the roadcut, as illustrated by the identified zone Fig. 11. Wedge failure is formed by the intersection of two independent planes that create a surface for a block(s) to slide on (Goodman, 1980). The intersection of the two planes forms a line that must dip steeper than the friction angle of the rockmass and less steep than the slope of the rockcut in order for kinematic failure to be possible, as illustrated by the identified zone in Fig. 11.

The ability to structurally map outcrop discontinuities using mobile terrestrial LiDAR data is an improvement in geomechanical evaluation technologies. The use of such data collection methodologies to enhance current geotechnical evaluation workflows will significantly reduce traditional field based evaluations, and hence reduce track delays and exposure of track engineers to the risk of evaluating hazardous rockcuts in the field. We now examine the use of mobile terrestrial LiDAR data in combination with other LiDAR data sources for more advanced applications. The RHRA focuses efforts into identifying high-risk areas. LiDAR data can be used to evaluate the identified areas where detailed kinematic and temporal analysis is required. 

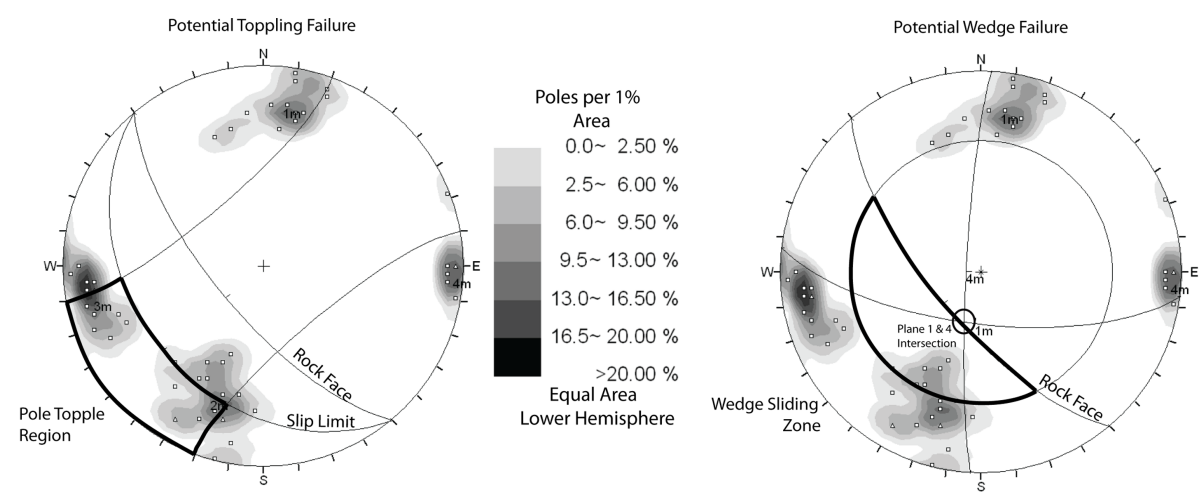

Fig. 11. Kinematic identification of possible rockfall failure modes present near mile 94 along the Algoma Central Railway. Topple and wedge failures are both identified as possible modes based on the LiDAR data analysis.

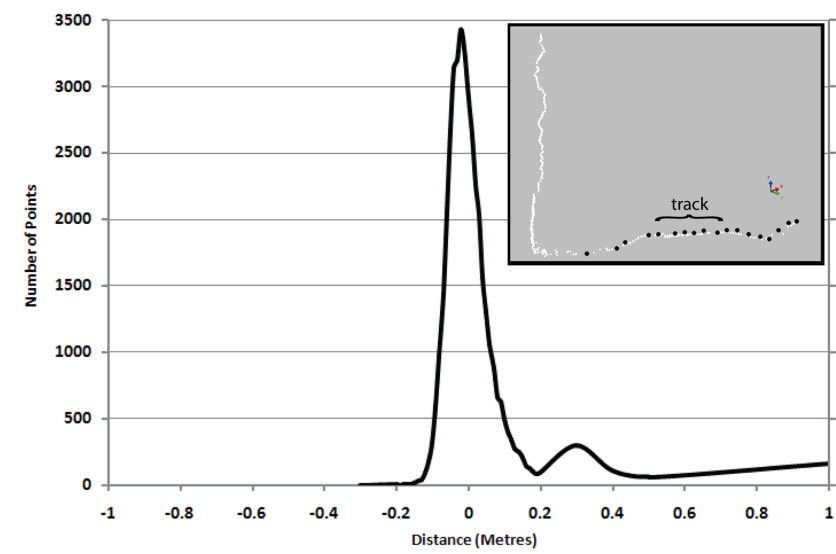

Fig. 12. Histogram illustrating the alignment comparison of TITAN data with helicopter data. The inset figure illustrates the alignment of the helicopter data (shown in black) with the titan data (shown in white). As well, illustrated in the inset figure is the general lack of helicopter data collected along the vertical rockface, due to the look-angle of the airborne laser. The two data sets are not manually aligned, rather they are simply viewed in a common space based on their individually collected geographic coordinate information.

\section{Data fusion}

TITAN data provides data ranging from track level to roughly $30 \mathrm{~m}$ beyond the track. Although the maximum scanning distance of TITAN is $200 \mathrm{~m}$, along the ACR there is a dense Boreal forest and numerous outcrops that limit the visibility of TITAN from the track outwards. There were however no instances where known track-side hazards were occluded in the TITAN dataset.

The ability to align and fuse TITAN data with other data sources will allow larger areas to be collected via airborne methodologies or detailed studies to be completed with highresolution static systems.

\subsection{Airborne and TITAN data: the ACR experiment, June 2008}

Aside from using TITAN along the ACR track, a "low-range" LiDAR system mounted on a helicopter was deployed to generate a valley scale LiDAR model. The low-range airborne LiDAR systems data were collected between 200$300 \mathrm{~m}$ above ground level. Low-range helicopter systems are designed to collect relatively dense data in comparison to fixed-wing aerial systems. This was completed during the summer of 2007, 12 months before the scanning of the ACR using the TITAN system. The resultant point-cloud produced by the helicopter system contains roughly $10 \mathrm{pts} / \mathrm{m}^{2}$. The airborne data were reported in two formats: un-edited and bareearth. A "bare earth" model consists of the points that are determined to lie directly on the earth's surface. Points representing trees, shrubs, and infrastructure, for example, are removed from the data set.

Fusion of the TITAN and Airborne bare earth data was achieved with the 3-D functionality of ArcGIS (ESRI, 2008). There was no adjustment of either dataset; they were simply aligned and merged based on their independently collected location information. For computing efficiency, only 1/12 of the data generated by TITAN were used for modelling completed in ArcGIS.

To determine the correlation between the TITAN and airborne data sets, an ArcGIS TIN model was built from the bare earth airborne data. Using the ArcGIS spot elevation function, the elevation on this TIN model at each TITAN point location was determined and then subtracted from the actual elevation recorded by the TITAN equipment. This operation was performed for a $1 \mathrm{~m}$ wide, $1 \mathrm{~km}$ long strip that roughly followed the centerline of the track. The results, illustrated by Fig. 12, show a good match between the datasets, with a distribution of height differences centred about a point about $1 \mathrm{~cm}$ below the airborne data (median $=-0.133 \mathrm{~m}$ ). The small bump around the $0.3 \mathrm{~m}$ division represents the ability of TITAN to accurately collect data from 


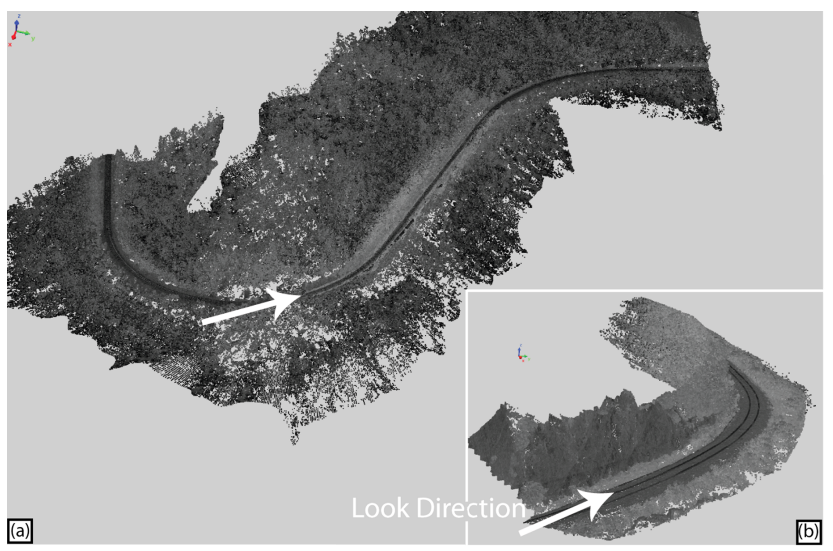

Fig. 13. (a) Raw TITAN data and raw airborne data collected along the ACR, the data is viewed in a common geographic space. (b) Raw TITAN data extracted from the combined model in Fig. 13a.

the surface of the rails along the track surface. $91 \%$ of the data lie within $\pm 10 \mathrm{~cm}$ and $96 \%$ within $\pm 15 \mathrm{~cm}$. The curve also shows a long positive tail likely caused by vegetation on the track and overhanging vegetation caught by the sideways and upward looking TITAN sensors. Once it was determined that the two datasets agreed vertically, they were merged into a single file. Vegetation was removed from the TITAN data by filtering any TITAN points that were more that $0.15 \mathrm{~m}$ above the bare-earth airborne data. A $7 \mathrm{~m}$ wide strip running parallel to the railway centerline was defined and the airborne data within this strip was replaced by the filtered TITAN data (Fig. 13).

This process was completed to establish that although the data were collected by different equipment, at different times, using different GPS-INS solutions, the data could be integrated for engineering purposes.

\subsection{Fusing TITAN and static data}

A segment of data collected by TITAN near Ottawa, Ontario was used to evaluate the fusion of high-resolution static data with TITAN data. The static data were not collected with GPS so the cloud had no global spatial properties. The data sets were aligned in true space using visible points of commonality and best-fit algorithms in the PolyWorks software package, allowing the high-resolution data to assume true coordinates based on the information extracted from TITAN. Figure 14 illustrates the alignment. Error mapping was completed to assess the alignment and the datasets line up with an average error of $\pm 3 \mathrm{~cm}$. This is not a simple or logical process because it involves the fusion of data collected at different times, different resolutions, and most challengingly - using different data formats.

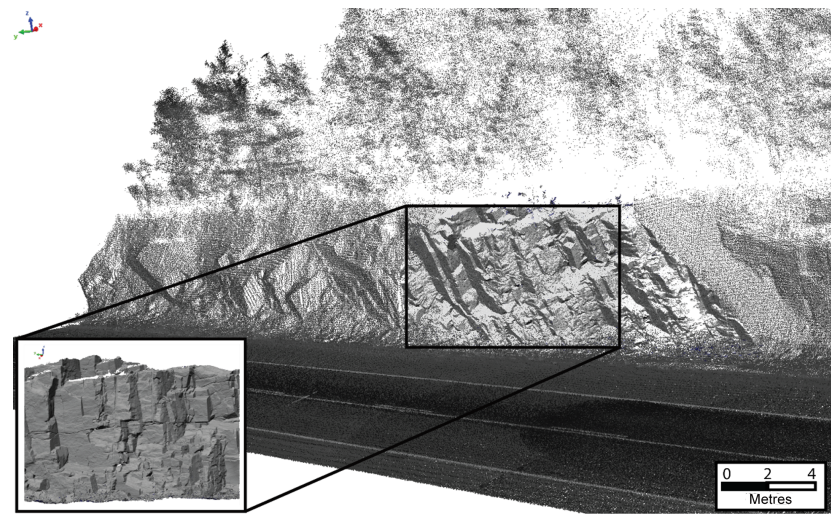

Fig. 14. Fusion of high-resolution Leica HDS 6000 data with mobile TITAN data collected near Ottawa, Ontario - resulting in the production of a locally precise and globally accurate point cloud.

\subsubsection{Difference monitoring}

The ability to fuse high resolution LiDAR data with TITAN allows the inherent qualities of the high-resolution data to be exploited. One significant advantage of high-resolution data (up to $10000 \mathrm{pts} / \mathrm{m}^{2}$ ) over TITAN data (practically up to $500 \mathrm{pts} / \mathrm{m}^{2}$ ) is the ability to conduct small scale differential monitoring. Differential monitoring requires successive scans to be completed evaluating the same area, effectively generating a 4-dimensional model (Donovan and Raza, 2008). Figure 15 illustrates an example of a rockcut that was scanned at two intervals. The first scan is the baseline data that will be used to evaluate successive scans for apparent movement or rock block release. Before the second scan was completed small rock blocks were manually removed from the face (hence only apparent differentiation is observed in the lower section of the outcrop, where rocks were safely accessible by the scanning team). The coulour ramp indicates distance from the nearest neighbor - comparing one scan to the next (grey indicates no apparent differential movement). The amount of differential movement observed in this example is between 3 and $15 \mathrm{~cm}$. The main assumption made in this analysis is that the tie-points used to align the successive scans are in the same position during both data collection periods. If the entire rock slope has moved tie-points located away from the rock face should be used.

These results conclude that differential monitoring using high-resolution LiDAR can enable the detection of small rock block release (sub $15 \mathrm{~cm}$ ). This is an extremely valuable tool for monitoring progressive failure in a given rockmass. TITAN data can be used for temporal modelling as long as the detectable movement is greater than the known combined global and local errors. This enables, in a best-case scenario, detection of roughly $30-50 \mathrm{~cm}$ edge length blocks. 


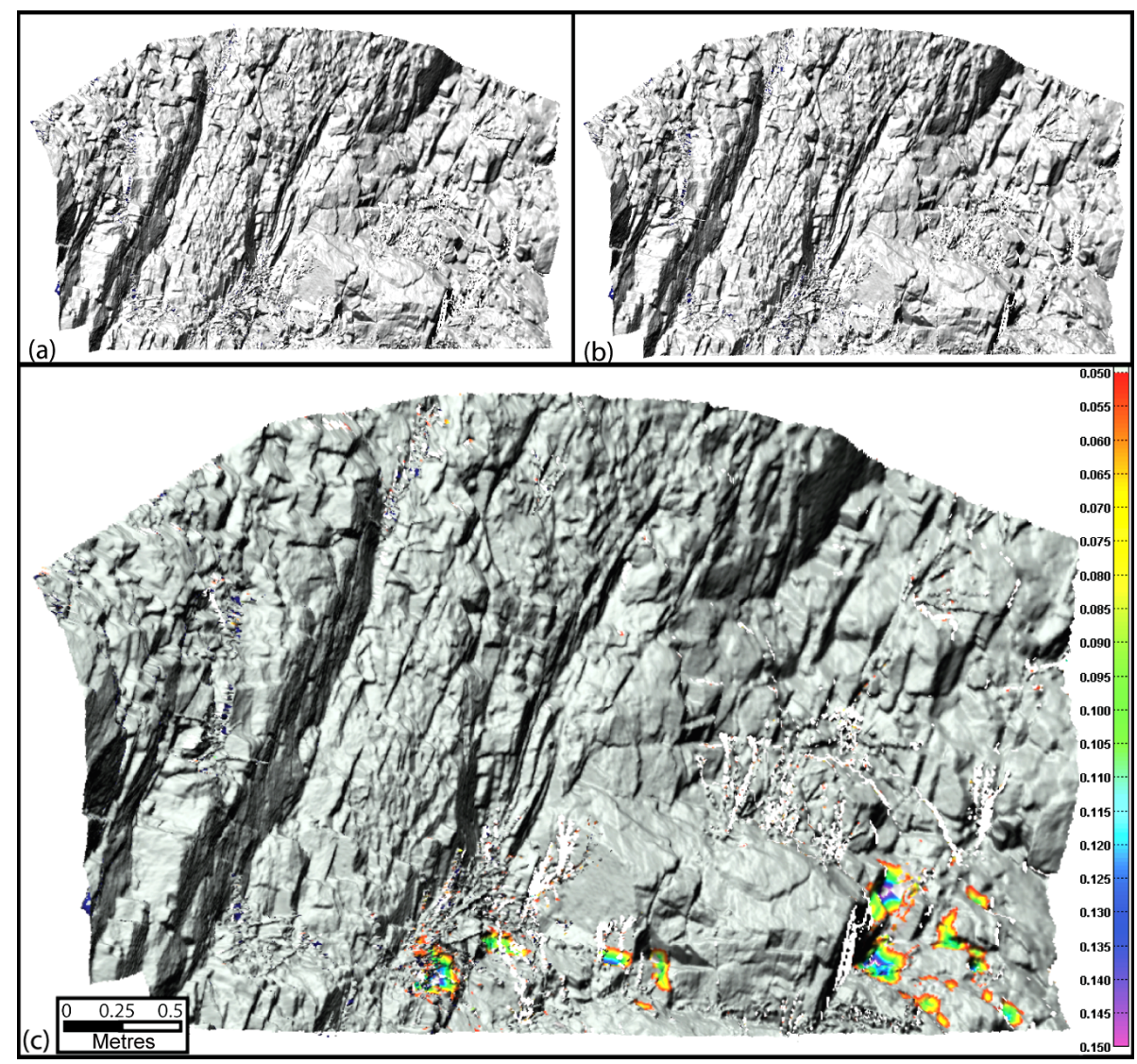

Fig. 15. Volumetric change detection by comparison of successive scans in time from a similar location. Identified zones represent between 5 and $10 \mathrm{~cm}$ of movement or release of rock blocks.

\section{Discussion}

The examples illustrated in this paper outline the use of mobile terrestrial and static LiDAR in comparison to traditional fieldwork completed by an experienced engineer. The data used in these analyses were from three sources: airborne, mobile terrestrial - TITAN, and high-resolution static equipment - Leica HDS 6000. The examples described involve the use of advanced technology, processing tools, and computational power. Through the use of these tools, many advantages of using LiDAR are readily identified.

Other engineering applications of mobile terrestrial $\mathrm{Li}$ DAR fused with airborne LiDAR are rockfall modelling and viewshed analyses. Rockfall models can be used to statistically evaluate potential rockfall paths and final positions. Traditional 2-D models are limited to a single possible profile of movement. 3-D models enable direct interaction of the falling rock with detailed topography, as illustrated by Lan et al. (2007).

Viewshed analyses enable the visible distance to be calculated from a known location, the potential visibility is controlled by topography. The quality of the topographic data used for this analysis in terms of density, accuracy, and coverage directly influence the result. The calculated visibility can be used to directly calculate stopping distances.

As workflows develop that incorporate the use of LiDAR data, regardless of the source, it will be critical that automation of feature extraction evolve as well. There needs to be better integration with GIS and CAD based programs. As well, data visualization would greatly benefit from the ability to colour the point cloud based on high-resolution photography.

As the price of LiDAR data collection decreases, positioning systems become more accurate, and processing tools become easier to use, the incorporation of mobile terrestrial LiDAR scanning into active rail usage will be a part of the general workflow. If trains are equipped with LiDAR scanners, real-time differential models could be generated as the train drives the rail line. This would generate a real-time hazard map identifying locations of mass-movement or the release of rock blocks.

The key to this work is not to speed up the operational process of identifying hazards but to increase the safety, accuracy, and repeatability of their identification by using new state-of-the-art tools. For example geo-simulation tools such as Train-MAGS (Mekni et al., 2008) can be used to 
dynamically calculate maximum train speed based on stopping distance. The most powerful and useful advantages are: data fusion, feature extraction, differential monitoring, rockfall analysis, and viewshed analysis.

\section{Conclusions}

The ability to use LiDAR data to identify and examine geomechanically controlled structural features has been demonstrated and proven for many applications. However, the innovative use of mobile terrestrial LiDAR for such applications demonstrates significant advantages over traditionally employed static terrestrial LiDAR including coverage, rate of acquisition, dynamic collection, and integration with corridor operation. Mobile terrestrial LiDAR is an evolving technology and the combination of tools available to extract and analyze features within the data are also evolving. The examples in the paper and those being researched by others will prove to be a cornerstone for a new direction with respect to geomechanical feature identification, especially for sites in remote areas and those along active transportation corridors.

Acknowledgements. The authors would like to thank Transport Canada, GEOIDE, NSERC, and the OCE for research funding. $\mathrm{CN}$ Rail provided track access and personnel during scanning.

Edited by: M.-H. Derron

Reviewed by: S. Buckley and another anonymous referee

\section{References}

Abbott, B., Bruce, I., Keegan, T., Oboni, F., and, Savigny, W.: A Methodology for the Assessment of Rockfall Hazard and Risk Along Linear Transportation Corridors, 8th Congress, International Assoc. Of Engineering Geology, A Global View from the Pacific Rim, Vancouver: Balkema, A. A.,Vancouver, British Columbia, 2, 1195-1200, 1998.

Alshawa, M., Smigiel, E., Grussenmeyer, P., and Landes, T.: Integration of a terrestrial LiDAR on a mobile mapping platform: first experiences, 5th International Symposium on Mobile Mapping Technology, Italy, p. 6, 2007.

Barber, D., Mills, J., and Smith-Voysey, S.: Geometric validation of a ground-based mobile laser scanning system, Journal of Photogrammetry and Remote Sensing, 63, 128-141, 2008.

Buckley, S. J., Howell, J. A., Enge, H. D., and Kurz, T. H.: Terrestrial laser scanning in geology: data acquisition, processing and accuracy considerations, J. Geol. Soc. London, 165, 625-638, 2008.

Donovan, J. and Raza, A. W.: A change detection method for slope monitoring and identification of potential rockfall using threedimensional imaging, San Francisco, American Rock Mechanics Association, X., 2008.

El-Rabbany, A.: Norwood: Artech House Publishers, Introduction to GPS: The Global Positioning System., 2nd Edition , 2006.

ESRI: ArcGIS V9.3 (Build 1770), Redlands, Ca. USA, 2008
Feng, Q. and Roshoff, K.: In situ mapping and documentation of rock fases using a full-coverage 3-D laser scanning technique, Paper 1A 23, CD-ROM, Int. J. Rock Mech. Min., 41(3), 379, 2004.

Glennie, C.: Reign of point clouds: a kinematic terrestrial LiDAR scanning system, InsideGNSS, Fall 2007, 21-31, 2007a.

Glennie, C.: Rigorous 3D error analysis of kinematic scanning LiDAR systems, J. Appl. Geodesy, 1, 147-157, 2007 b.

Goodman, R. E.: Introduction to rock mechanicsm, edited by: Wiley, J., Toronto, Chapter 8, 1980.

InnovMetric: PolyWorks V10.1, Quebec City, Qu., Canada, 2008.

Lan, H., Martin, C. D., and Lim, C. H.: Rockfall Analyst: a GIS extension for three-dimensional and spatially distributed rockfall hazard monitoring, Comput. Geosci., 33, 262-279, 2007.

Lato, M., Hutchinson, D. J., Diederichs, M. S., and Harrap, R.: Optimization of LiDAR scanning and processing for automated structural evaluation of discontinuities in rockmasses, Int. J. Rock Mech. Min., 46, 194-199, 2009.

Maerz, N. H.: Highway rock cut stability assessment in rock massed not conducive to stability calculations, Proceedings of the 51st Annual Highway Geology Symposium, Seattle, Washington, 249-259, 2000.

Mason, D. C., Horritt, M. S., Hunter, N. M., and Bates, P. D.: Use of fused airborne scanning laser altimetry and digital map data for urban flood modelling, Hydrol. Process., 21(11), 1436-1447, 2007.

McKean, J. and Roering, J.: Objective landlside detection and surface morphology mapping using high-resolustion airborne laser altimetre, Geomorphology, 57, 331-351, 2004.

Mekni, M., Sahli, N., and Moulin, B.: A geosimulation approach involving spatially-aware agents: A case study on the identification of risky areas for trains, SpringSim, 1-56555-319-5, 37-45, 2008.

Optech. Inc. , Lynx Mobile Mapper, Toronto, On. Canada 2008.

Pritchard, M., Porter, M., Savigny, K. W., Bruce, I., Oboni, F., Keegan, T., et al.: $\mathrm{CN}$ rockfall hazard risk management system: experience, enhancements, and future direction, The American Railway Engineering and Maintenance-of-Way Association, Chicago, 2005.

RocScience, Dips 5.1. Toronto, On. Canada, 2005.

Rosser, N., Dunning, S., Lim, M., and Petley, D.: Terrestrial laser scanning for quantative rockfall hazard assessment, in: Landslide risk management, edited by: Hunger, O., Fell, R., Couture, R., Eberhardt, E., Rotterdam, Balkema, p. 091, 2005.

Staley, D. M., Wasklewick, T. A., and Blaszcynski, J. S.: Surficial patterns of debris flow deposition on alluvial fans in Death Valley, CA using airborne laser swath mapping data, Geomorphology, 74(1-4), 152-163, 2005.

Sturzenegger, M., Stead, D., Froese, C., Moreno, F., and Jaboyedoff, M.: Ground-based and airborne LiDAR for structural mapping of a large landslide: the Frank Slide, in: Proceedings of the first Canadian US rock mechanics symposium vol. 2, edited by: Eberhardt, E., Stead, D., and, Morrison, T., Vancouver, London, Taylor and Francis, 925-932, 2007a.

Sturzenegger, M., Yan, M., Stead, D., and Elmo, D.: Applications and limitiations of ground-based laser scanning in rock slope characterization, in: Proceedings of the first Canadian US rock mechanics symposium vol. 1, edited by: Eberhardt, E., Stead, D. and, Morrison, T., London, Taylor and Francis, 29-36, $2007 \mathrm{~b}$. 
Turner, A. K., Kemeny, J., Slob, S., and Hack, R.: Evaluation and management of unstable rock slopes by 3-D laser scanning, Internation Association for Engineering Geology and the Environmnet, paper no. 404, Geol. Soc. London, 1-11, 2006.
Yang, Y. and Farrell, J. A.: Magnetometer and differential carrier phase GPS-aided INS for advanced vehicle control, IEEE T. Robotic. Autom., 19(2), 269-282, 2003. 